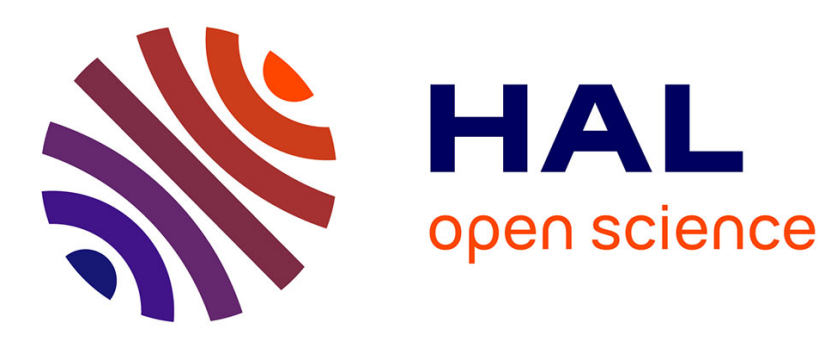

\title{
On the study of faster-than-Nyquist multicarrier signaling based on frame theory
}

Cyrille Siclet, Damien Roque, Huaqiang Shu, Pierre Siohan

\section{To cite this version:}

Cyrille Siclet, Damien Roque, Huaqiang Shu, Pierre Siohan. On the study of faster-than-Nyquist multicarrier signaling based on frame theory. ISWCS 2014 - 11th International Symposium on Wireless Communication Systems, Aug 2014, Barcelone, Spain. 10.1109/ISWCS.2014.6933356 hal-01260509

\section{HAL Id: hal-01260509 \\ https://hal.science/hal-01260509}

Submitted on 4 Feb 2016

HAL is a multi-disciplinary open access archive for the deposit and dissemination of scientific research documents, whether they are published or not. The documents may come from teaching and research institutions in France or abroad, or from public or private research centers.
L'archive ouverte pluridisciplinaire HAL, est destinée au dépôt et à la diffusion de documents scientifiques de niveau recherche, publiés ou non, émanant des établissements d'enseignement et de recherche français ou étrangers, des laboratoires publics ou privés. 


\title{
On the Study of Faster-than-Nyquist Multicarrier Signaling Based on Frame Theory
}

\author{
Cyrille Siclet*, Damien Roque ${ }^{\dagger}$, Huaqiang Shu* and Pierre Siohan ${ }^{\ddagger}$ \\ *Grenoble Images Parole Signal Automatique (GIPSA-lab) \\ 38402 Grenoble, FRANCE \\ Email: \{cyrille.siclet,huaqiang.shu\}@gipsa-lab.grenoble-inp.fr \\ ${ }^{\dagger}$ University of Toulouse, ISAE \\ 31055 Toulouse, FRANCE \\ Email: damien.roque@isae.fr \\ $\ddagger$ Orange-Labs \\ 35512 Cesson Sévigné, FRANCE \\ Email: pierre.siohan@orange.com
}

\begin{abstract}
Multicarrier transmissions are classically based on undercomplete or exact Weyl-Heisenberg Riesz (biorthogonal or orthogonal) bases implemented thanks to oversampled filterbanks. This can be seen as a transmission below the Nyquist rate. However, when overcomplete Weyl-Heisenberg frames are used, we obtain a "faster-than-Nyquist" (FTN) system and it is theoretically impossible to recover exactly transmitted symbols using a linear receiver. Various studies have shown the interest of this high density signaling scheme as well as practical implementations based on trellis and/or iterative decoding. Nevertheless, there is still a lack of theoretical justifications with regard to pulse design in the FTN case. In this paper, we consider a linear transceiver operating over an additive white Gaussian noise channel. Using the frame theory and simulation results, we show that the mean squared error (MSE) is minimized when tight frames are used.
\end{abstract}

\section{INTRODUCTION}

It is usually recognized that perfect reconstruction of the symbols is one of the necessary conditions for reliable information transmission. This constraint implies biorthogonal synthesis and analysis bases (used respectively by the transmitter and the receiver). In the particular case of $T$-spaced waveforms, it leads to the Nyquist inter-symbol interference (ISI) free criterion [1]. This strategy has the advantage to allow a low-complexity optimal detector (linear receiver) at the expense of a maximum signaling density: the bandwidth has to be greater or equal than $1 / T$.

With an increasing need for spectral efficiency, it has been suggested to override the Nyquist criterion leading to "faster-than-Nyquist" (FTN) transceivers. The first approach developed by J. Mazo was based on an orthogonal single carrier system with cardinal sine pulses and binary antipodal symbols [2]. From this setup, he showed that an increase of the signaling rate up to $25 \%$ has no consequence on the minimum euclidean distance between sequences of symbols. According to G.D. Forney's optimal detection over ISI channels, it follows from this result that error probability can remain unchanged even if the receiver's complexity is increased [3].

Three decades later, F. Rusek et al. provided a generalization of FTN transmission to non-binary alphabets with an emphasis on reconstruction techniques [4]. Simplified trellis detector as well as iterative decoders have been proposed in order to target practical implementations [5], [6]. It confirms that binaryerror-rate (BER) over additive white Gaussian noise (AWGN) channels are similar to orthogonal systems when the density is increased up to $30 \%$.

When facing time-frequency selective channels, multicarrier modulations are usually justified since they allow to distribute information in the time-frequency plane using appropriate pulse shapes [7], [8]. However, in the context of a FTN linear system with limited output power, the challenge is to determine appropriate time-frequency spacing and pulse shaping in order to reach a trade-off between throughput, bandwidth and reconstruction error. If one further assumes a fixed bandwidth, it follows that channel-induced interference mitigation is tied to the signaling density.

Despites attractive practical implementations of FTN multicarrier systems [9], [10], a lack of theoretical justifications appears concerning their design. Such systems are usually derived from (bi)orthogonal transceivers where higher density time-frequency lattices are used. In this paper, we restrict our study to linear transceivers and focus on the minimization of the normalized mean squared error (NMSE) in presence of inter-pulse interference and additive white Gaussian noise. Our main contributions include an analysis based on the frame theory as well as practical guidelines for FTN multicarrier modulations design.

This paper is organized as follows. After presenting notations and definitions used throughout the document, section 2 describes the multicarrier transceiver based on WeylHeisenberg families. In this context, a general definition of FTN signaling is given based on the density of its timefrequency lattice. Section 3 discusses the design of the pulse shapes in order to minimize the MSE introduced by a linear transceiver operating over an AWGN channel. Section 4 confirms the theoretical study based on simulation results. Finally, conclusions are presented in section 5 . 


\section{System Model}

In this section, we first present our notations and recall basic definitions from the frame theory. Afterwards, we establish the input-output relations of a linear multicarrier transceiver in a faster-than-Nyquist context.

\section{A. Notations and definitions}

Let $\mathbf{V}$ be an Hilbert space. For two vectors $x, y \in \mathbf{V}$, the inner product is denoted $\langle x, y\rangle$ and the norm of $x$ is given by $\|x\|=\sqrt{\langle x, x\rangle}$. In particular, we define for $p \in\{1,2\}$ the spaces $\mathcal{L}_{p}(\mathbf{R})=\left\{x: \mathbf{R} \rightarrow \mathbf{C} / \int_{-\infty}^{+\infty}|x(t)|^{p} \mathrm{~d} t<+\infty\right\}$ and $\ell_{p}(\mathbf{I})=\left\{x: \mathbf{I} \rightarrow \mathbf{C} / \sum_{k \in \mathbf{I}}|x[k]|^{p}<+\infty\right\}$ with $\mathbf{I}$ a countable index set.

Let us define $\boldsymbol{f}=\left\{f_{k}\right\}_{k \in \mathbf{I}}$ a family of vectors in $\mathbf{V}$. We say that $\boldsymbol{f}$ is a frame of $\mathbf{V}$ if there exist two constants $0<$ $A \leq B<+\infty$ such that

$$
A\|f\|^{2} \leq \sum_{k \in \mathbf{I}}\left|\left\langle f, f_{k}\right\rangle\right|^{2} \leq B\|f\|^{2}, \quad \forall f \in \mathbf{V} .
$$

The constants $A, B$ are called lower and upper frame bounds (respectively) for the frame. If $A=B, \boldsymbol{f}$ is a tight frame. Let us define $\boldsymbol{g}=\left\{g_{k}\right\}_{k \in \mathbf{I}}$ another frame of $\mathbf{V}$; we say that $\boldsymbol{f}$ and $\boldsymbol{g}$ are dual frames if and only if

$$
f=\sum_{k \in \mathbf{I}}\left\langle g_{k}, f\right\rangle f_{k}, \quad \forall f \in \mathbf{V} .
$$

If there exist not all zero coefficients $\left\{c_{k}\right\}_{k \in \mathbf{I}} \subset \ell_{2}(\mathbf{I})$ such that $\sum_{k \in \mathbf{I}} c_{k} f_{k}=0$ then the frame is overcomplete and several dual frames exist [11, p. 107]. Conversely, if $\sum_{k \in \mathbf{I}} c_{k} f_{k}=0$ only if $c_{k}=0, \forall k \in \mathbf{I}$, the frame $\boldsymbol{f}$ is a Riesz basis and a unique dual frame exists.

In order to introduce compact notations related to frame operations, we define the synthesis and analysis operators $\mathrm{D}_{\boldsymbol{f}}$ and $\mathrm{D}_{f}^{*}$ associated to $f$ :

$$
\begin{aligned}
& \mathrm{D}_{\boldsymbol{f}}: \ell_{2}(\mathbf{I}) \rightarrow \mathbf{V},\left\{c_{k}\right\}_{k \in \mathbf{I}} \mapsto \sum_{k \in \mathbf{I}} c_{k} f_{k}, \\
& \mathrm{D}_{\boldsymbol{f}}^{*}: \mathbf{V} \rightarrow \ell_{2}(\mathbf{I}), f \mapsto\left\{\left\langle f_{k}, f\right\rangle\right\}_{k \in \mathbf{I}}
\end{aligned}
$$

where ${ }^{*}$ is the adjoint operator. It is then possible to define the frame operator $\mathrm{S}$ based on successive analysis and synthesis:

$$
\mathrm{S}_{\boldsymbol{f}} f=\mathrm{D}_{\boldsymbol{f}} \mathrm{D}_{\boldsymbol{f}}^{*} f=\sum_{k \in \mathbf{I}}\left\langle f_{k}, f\right\rangle f_{k}
$$

Notice that $\mathrm{S}$ is bounded, invertible and self-adjoint. It follows that $\tilde{\boldsymbol{f}}=\left\{\tilde{f}_{k}=\mathrm{S}_{\boldsymbol{f}}^{-1} f_{k}\right\}_{k \in \mathbf{I}}$ is also a frame with bounds $1 / B$ and $1 / A[11$, p. 100$]$. The frame $\tilde{f}$ is called the canonical dual frame of $\boldsymbol{f}$ and it verifies the following property: among all scalar sequences $\left\{c_{k}\right\}_{k \in \mathbf{I}}$ for which $f=\sum_{k \in \mathbf{I}} c_{k} f_{k}$, the coefficient sequence $\left\{\left\langle f, \tilde{f}_{k}\right\rangle\right\}_{k \in \mathbf{I}}$ has minimal $\ell_{2}$-norm [11, p. $6]$.

With the aim of representing multicarrier systems, it is convenient to introduce the particular class of Weyl-Heisenberg frames (sometimes called Gabor frames) [12]. We start by defining translation and modulation operators, denoted respectively $\mathrm{T}_{T_{0}}$ and $\mathrm{M}_{F_{0}}$, with $F_{0}, T_{0} \in \mathbf{R}$ :

$$
\begin{aligned}
& \mathrm{M}_{F_{0}}: \mathcal{L}_{2}(\mathbf{R}) \rightarrow \mathcal{L}_{2}(\mathbf{R}), f(t) \mapsto f(t) e^{j 2 \pi F_{0} t}, \\
& \mathrm{~T}_{T_{0}}: \mathcal{L}_{2}(\mathbf{R}) \rightarrow \mathcal{L}_{2}(\mathbf{R}), f(t) \mapsto f\left(t-T_{0}\right) .
\end{aligned}
$$

We consider a lattice $\left\{m F_{0}, n T_{0}\right\}_{(m, n) \in \boldsymbol{\Lambda}} \subset \mathbf{R}^{2}$ with $\boldsymbol{\Lambda} \subset$ $\mathbf{Z}^{2}$; its density is denoted $\rho=1 /\left(F_{0} T_{0}\right)$. The family of regularly modulated and translated function $g \in \mathcal{L}_{2}(\mathbf{R})$ is written as $\boldsymbol{g}=\left\{\mathrm{M}_{m F_{0}} \mathrm{~T}_{n T_{0}} g\right\}_{(m, n) \in \boldsymbol{\Lambda}}$; it forms a WeylHeisenberg frame if it fulfills (1).

\section{B. Back-to-back input-output relation}

At the transmitter side, let us define a sequence of complex coefficients $\boldsymbol{c}=\left\{c_{m, n}\right\}_{(m, n) \in \boldsymbol{\Lambda}} \in \ell_{2}(\boldsymbol{\Lambda})$ with $\boldsymbol{\Lambda} \subset \mathbf{Z}^{2}$. Let $\boldsymbol{g}=\left\{g_{m, n}\right\}_{(m, n) \in \boldsymbol{\Lambda}}$ be a Weyl-Heisenberg frame with parameters $F_{0}, T_{0}>0$, bounds $0<A \leq B<+\infty$ and whose vectors are derived from a generator $g(t) \in \mathcal{L}_{2}(\mathbf{R})$ :

$$
g_{m, n}(t)=g\left(t-n T_{0}\right) e^{j 2 \pi m F_{0} t} .
$$

The signal $s(t)$ at the output of the multicarrier transmitter can be written as a synthesis operation based on the sequence $\boldsymbol{c}$ and the Weyl-Heisenberg family $\boldsymbol{g}$ :

$$
s(t)=\left(\mathrm{D}_{\boldsymbol{g}} \boldsymbol{c}\right)(t)=\sum_{(m, n) \in \boldsymbol{\Lambda}} c_{m, n} g_{m, n}(t), \quad t \in \mathbf{R} .
$$

As a result of this expansion, information carried by the complex symbol sequence is regularly spread in the timefrequency plane (fig. 1) with a minimum distance $T_{0}$ in time and $F_{0}$ in frequency.

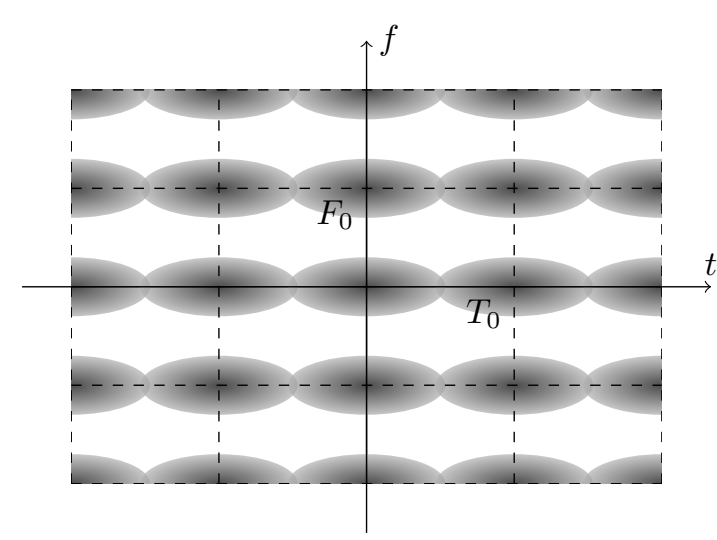

Fig. 1: Representation of transmitted signal energy in the timefrequency plane. In this example, a Gaussian-shaped generator function $g$ is used and the lattice parameters allow a clear separation between pulses in the frequency domain and an overlap in the time domain.

At the receiver side, we consider a frame $\check{\boldsymbol{g}}=\left\{\check{g}_{p, q}\right\}_{(p, q) \in \boldsymbol{\Lambda}}$ with bounds $0<\check{A} \leq \check{B}<+\infty$. A linearly estimated sequence of coefficients $\hat{\boldsymbol{c}}=\left\{\hat{c}_{p, q}\right\}_{(p, q) \in \boldsymbol{\Lambda}}$ is obtained by an analysis operation based on the received signal $r(t)$ and the analysis frame $\check{\boldsymbol{g}}$ :

$$
\hat{\boldsymbol{c}}=\left\{\hat{c}_{p, q}\right\}_{(p, q) \in \boldsymbol{\Lambda}}=\mathrm{D}_{\check{\boldsymbol{g}}}^{*} r=\left\{\left\langle\check{g}_{p, q}, r\right\rangle\right\}_{(p, q) \in \boldsymbol{\Lambda}} .
$$


The convergence of the double sum mentioned in (9) is guaranteed by the upper bound of $\boldsymbol{g}$ and ensures a stable synthesis. Moreover, the lower bound of the frame also ensures that a non-zero input sequence generates a non-zero transmitted signal. Conversely the frame structure of $\check{\boldsymbol{g}}$ guarantees the stability of the analysis operation specified in (10).

We first consider a perfect channel, i.e. $r(t)=s(t)$, such that an estimated symbol $\hat{c}_{p, q}$, with $(p, q) \in \boldsymbol{\Lambda}$ is given by

$$
\hat{c}_{p, q}=\left\langle\check{g}_{p, q}, s\right\rangle=\sum_{(m, n) \in \boldsymbol{\Lambda}} c_{m, n}\left\langle\check{g}_{p, q}, g_{m, n}\right\rangle
$$

For brevity, we define $\boldsymbol{\Lambda}_{\mathbf{p}, \mathbf{q}}=\boldsymbol{\Lambda} \backslash\{(p, q)\}$ and we rewrite (11) in order to identify a useful signal term and an interference term:

$$
\hat{c}_{p, q}=\underbrace{c_{p, q}\left\langle\check{g}_{p, q}, g_{p, q}\right\rangle}_{\text {useful signal }}+\underbrace{\sum_{(m, n) \in \Lambda_{\mathbf{p}, \mathbf{q}}} c_{m, n}\left\langle\check{g}_{p, q}, g_{m, n}\right\rangle}_{\text {interference }} .
$$

In order to recover the transmitted symbols without ambiguity (i.e. with no interference), it is necessary to choose $\boldsymbol{g}$ and $\check{\boldsymbol{g}}$ as biorthogonal Riesz sequences. This constraint requires $\rho \leq 1$ which limits the signaling density in the time-frequency plane. In this study, we consider faster-than-Nyquist transceivers that fulfill the relation $\rho>1$. A consequence of this high signaling density prevents $\boldsymbol{g}$ to be a Riesz sequence. It follows that biorthogonality can't be obtained, leading to inter-pulse interference.

The FTN constraint raises several questions for the multicarrier transceiver design. For a given time-frequency lattice, how to choose $\check{g}$ in order to minimize the interference and noise power? Are there practical methods to derive $\check{\boldsymbol{g}}$ from $\boldsymbol{g}$ ? The following section discusses these questions using the frame theory.

\section{NMSE STUDY IN PRESENCE OF INTERFERENCE AND NOISE}

In this section, we develop the conditions for NMSE minimization over an AWGN channel. We assume a given timefrequency lattice with parameters $T_{0}, F_{0}>0$ and a generator function $g(t) \in \mathcal{L}_{2}(\mathbf{R})$. In a more realistic scenario, these parameters would be determined with regard to the time and frequency selectivity introduced by the channel, considering a target signal-to-interference ratio or signaling density. Such considerations are explained in [7], [8] with $\rho \geq 1$ and won't be discussed in the following. The noise is denoted $z(t)$ such that the received signal is $r(t)=s(t)+z(t)$ and the estimated coefficients are $\hat{c}_{p, q}=\left\langle\check{g}_{p, q}, s\right\rangle+\left\langle\check{g}_{p, q}, z\right\rangle$.

The symbols are assumed zero-mean, independent and with same variance $\sigma_{c}^{2}$. The noise $z(t)$ is a centered complex Gaussian process, independent of the symbols, characterized by its power spectral density $\sigma_{z}^{2} / W$ for $|f| \leq W / 2$ and 0 otherwise (with $0<W<+\infty$ ). Let us define the received normalized symbol $\bar{c}_{p, q}$ with $(p, q) \in \boldsymbol{\Lambda}$

$$
\begin{aligned}
& \bar{c}_{p, q}=\frac{\hat{c}_{p, q}}{\left\langle\check{g}_{p, q}, g_{p, q}\right\rangle} \\
& =c_{p, q}+\underbrace{\sum_{(m, n) \in \Lambda_{\mathbf{p}, \mathbf{q}}} c_{m, n} \frac{\left\langle\check{g}_{p, q}, g_{m, n}\right\rangle}{\left\langle\check{g}_{p, q}, g_{p, q}\right\rangle}}_{i_{p, q}}+\underbrace{\frac{\left\langle\check{g}_{p, q}, z\right\rangle}{\left\langle\check{g}_{p, q}, g_{p, q}\right\rangle}}_{z_{p, q}}
\end{aligned}
$$

Denoting $\mathrm{E}(\cdot)$ the expected value operator, the NMSE of the received symbol can be expressed as:

$$
\begin{aligned}
& \frac{\mathrm{E}\left(\left|\bar{c}_{p, q}-c_{p, q}\right|^{2}\right)}{\sigma_{c}^{2}}= \\
& \underbrace{\sum_{(m, n) \in \Lambda_{\mathbf{p}, \mathbf{q}}} \frac{\left|\left\langle\check{g}_{p, q}, g_{m, n}\right\rangle\right|^{2}}{\left|\left\langle\check{g}_{p, q}, g_{p, q}\right\rangle\right|^{2}}}_{\sigma_{i_{p, q}^{2}}^{2} / \sigma_{c}^{2}}+\underbrace{\frac{\left\|\check{g}_{p, q}\right\|^{2}}{\left|\left\langle\check{g}_{p, q}, g_{p, q}\right\rangle\right|^{2}} \frac{\sigma_{z}^{2}}{\sigma_{c}^{2}}}_{\sigma_{z_{p, q}}^{2} / \sigma_{c}^{2}}
\end{aligned}
$$

Finally, the NMSE splits into two parts: the first, $\sigma_{i_{p, q}}^{2} / \sigma_{c}^{2}$ ties to the inter-pulse-interference and the second, $\sigma_{z_{p, q}}^{2} / \sigma_{c}^{2}$ is related to the noise. In the following, we discuss the separated minimization of both terms under the constraint of a non-null estimated symbol energy.

\section{A. Interference term minimization}

In view of (14) and according to [11, lemma 5.3.6], the minimization of the $\ell_{2}$-norm of $\left\{\left\langle\check{g}_{p, q}, g_{m, n}\right\rangle\right\}_{(m, n) \in \boldsymbol{\Lambda}_{\mathbf{p}, \mathbf{q}}}$ requires $\boldsymbol{g}$ and $\check{\boldsymbol{g}}$ to be canonical dual frames, that is $\left\{\check{g}_{m, n}=\right.$ $\left.\mathrm{S}^{-1} g_{m, n}\right\}_{(m, n) \in \boldsymbol{\Lambda}_{\mathbf{p}, \mathbf{q}}}$ which implies $\check{A}=1 / B$ and $\check{B}=1 / A$.

Since $\boldsymbol{g}$ has a Weyl-Heisenberg structure, it is useful to notice that $\mathrm{SM}_{m F_{0}} \mathrm{~T}_{n T_{0}}=\mathrm{M}_{m F_{0}} \mathrm{~T}_{n T_{0}} \mathrm{~S}$ and $\mathrm{S}^{-1} \mathrm{M}_{m F_{0}} \mathrm{~T}_{n T_{0}}=$ $\mathrm{M}_{m F_{0}} \mathrm{~T}_{n T_{0}} \mathrm{~S}^{-1}$ [11, lemma 9.3.1]. As a consequence, $\boldsymbol{g}$ and $\check{\boldsymbol{g}}$ share the same time-frequency lattice:

$$
\check{g}_{p, q}(t)=\check{g}\left(t-q T_{0}\right) e^{j 2 \pi p F_{0} t} .
$$

This important simplification means that it is enough to find $\check{g}=\mathrm{S}^{-1} g$ in order to derive the canonical dual frame of $\boldsymbol{g}$ [11, theorem 9.3.2]. Moreover, in this case, $\left|\left\langle\check{g}_{p, q}, g_{m, n}\right\rangle\right|=$ $\left|\left\langle\check{g}, g_{m-p, n-q}\right\rangle\right|$ such that

$$
\begin{aligned}
\frac{\sigma_{i_{p, q}}^{2}}{\sigma_{c}^{2}} & =\sum_{(m, n) \in \Lambda_{\mathbf{p}, \mathbf{q}}} \frac{\left|\left\langle\check{g}, g_{m-p, n-q}\right\rangle\right|^{2}}{|\langle\check{g}, g\rangle|^{2}} \\
\frac{\sigma_{z_{p, q}}^{2}}{\sigma_{c}^{2}} & =\frac{\|\check{g}\|^{2}}{|\langle\check{g}, g\rangle|^{2}} \frac{\sigma_{z}^{2}}{\sigma_{c}^{2}}
\end{aligned}
$$

In the following, we assume that $\boldsymbol{g}$ and $\check{\boldsymbol{g}}$ are canonical dual frames.

\section{B. Noise term minimization}

Then, the Cauchy-Schwartz inequality yields $|\langle\check{g}, g\rangle| \leq$ $\|\check{g}\|\|g\|$ or equivalently

$$
\frac{\|\check{g}\|^{2}}{|\langle\check{g}, g\rangle|^{2}} \geq \frac{1}{\|g\|^{2}} .
$$

The equality is satisfied when $g$ and $\check{g}$ are proportional, which means that $\boldsymbol{g}$ and $\check{\boldsymbol{g}}$ are tight Weyl-Heisenberg frames that 
fulfill the relation $\check{g}=(1 / A) g$. In this case assumed hereafter, $A=B$ and we obtain from (16) and (17)

$$
\begin{aligned}
\frac{\sigma_{i_{p, q}}^{2}}{\sigma_{c}^{2}} & =\sum_{(m, n) \in \boldsymbol{\Lambda}_{\mathbf{p}, \mathbf{q}}} \frac{\frac{1}{A^{2}}\left|\left\langle g, g_{m-p, n-q}\right\rangle\right|^{2}}{\frac{1}{A^{2}}|\langle g, g\rangle|^{2}} \\
& =\sum_{(m, n) \in \boldsymbol{\Lambda}_{\mathbf{p}, \mathbf{q}}} \frac{\left|\left\langle g, g_{m-p, n-q}\right\rangle\right|^{2}}{|\langle g, g\rangle|^{2}} \\
\frac{\sigma_{z_{p, q}}^{2}}{\sigma_{c}^{2}} & =\frac{\frac{1}{A^{2}}\|g\|^{2}}{\frac{1}{A^{2}}|\langle g, g\rangle|^{2}} \frac{\sigma_{z}^{2}}{\sigma_{c}^{2}}=\frac{\|g\|^{2}}{|\langle g, g\rangle|^{2}} \frac{\sigma_{z}^{2}}{\sigma_{c}^{2}}
\end{aligned}
$$

Furthermore, the tight frame equality yields

$$
\sum_{(m, n) \in \boldsymbol{\Lambda}}\left|\left\langle f, g_{m, n}\right\rangle\right|^{2}=A\|f\|^{2}, \quad \forall f \in \mathcal{L}_{2}(\mathbf{R}) .
$$

Particularly, for $f=g$, we have

$$
\begin{aligned}
\frac{\sigma_{i_{p, q}}^{2}}{\sigma_{c}^{2}} & =\frac{A\|g\|^{2}-\|g\|^{4}}{\|g\|^{4}}=\frac{A-\|g\|^{2}}{\|g\|^{2}} \\
\frac{\sigma_{z_{p, q}}^{2}}{\sigma_{c}^{2}} & =\frac{1}{\|g\|^{2}} \frac{\sigma_{z}^{2}}{\sigma_{c}^{2}} .
\end{aligned}
$$

\section{On the derivation of $\|g\|^{2}$}

According to the Wexler-Raz duality theorem [11, theorem 9.3.4], $\boldsymbol{g}=\left\{\mathrm{M}_{m F_{0}} \mathrm{~T}_{n T_{0}} g\right\}_{(m, n) \in \boldsymbol{\Lambda}}$ is a tight WeylHeisenberg frame with bound $A$ if and only if $\tilde{\boldsymbol{g}}=$ $\left\{\mathrm{M}_{m / T_{0}} \mathrm{~T}_{n / F_{0}} g\right\}_{(m, n) \in \Lambda}$ is an orthogonal family with bound $A / \rho$ :

$\left\langle g\left(t-\frac{n}{F_{0}}\right) e^{j 2 \pi m \frac{t}{T_{0}}}, g\left(t-\frac{q}{F_{0}}\right) e^{j 2 \pi p \frac{t}{T_{0}}}\right\rangle=\frac{A}{\rho} \delta_{m, p} \delta_{n, q}$.

Particularly, for $m=p=0$ and $n=q=0$, we obtain

$$
\langle g, g\rangle=\|g\|^{2}=\frac{A}{\rho} .
$$

Finally the theoretical interference and noise terms are obtained:

$$
\frac{\sigma_{i_{p, q}}^{2}}{\sigma_{c}^{2}}=\frac{1-F_{0} T_{0}}{F_{0} T_{0}}=\rho-1 \text { and } \frac{\sigma_{z_{p, q}}^{2}}{\sigma_{c}^{2}}=\frac{\rho}{A} \frac{\sigma_{z}^{2}}{\sigma_{c}^{2}} .
$$

This result confirms that interference and noise power increases with the signaling density $\rho$.

There are two ways to normalize the system:

- by imposing $A$, which yields $\|g\|=\sqrt{A / \rho}$;

- by imposing $\|g\|$, which yields $A=\rho\|g\|^{2}$ and $\check{g}=$ $g /\left(\rho\|g\|^{2}\right)$.

Particularly the case $A=1$ implies that the transmitter and the receiver share the same generator function $\check{g}=g$ with $\|g\|=1 / \sqrt{\rho}$. Moreover, the symbol energy is preserved over transmission, since for a tight frame, we have:

$$
\|s\|^{2}=A \sum_{(m, n) \in \boldsymbol{\Lambda}}\left|c_{m, n}\right|^{2}=\sum_{(m, n) \in \boldsymbol{\Lambda}}\left|c_{m, n}\right|^{2} .
$$

These results can be summarized in the following theorem.

Theorem 1: A multicarrier linear transceiver based on Weyl-Heisenberg families with density $\rho>1$ achieves a minimal mean squared error with AWGN channel when it uses a tight Weyl-Heseinberg frame for transmission and reception. Moreover the NMSE is then given by

$$
\frac{\mathrm{E}\left(\left|\bar{c}_{p, q}-c_{p, q}\right|^{2}\right)}{\sigma_{c}^{2}}=(\rho-1)+\frac{\rho}{A} \frac{\sigma_{z}^{2}}{\sigma_{c}^{2}}
$$

\section{Simulations Under AWGN CHANNEL}

In this section, we measure the performances of various FTN multicarrier systems in terms of NMSE. The linear transceiver is characterized by a number of subcarriers $M=$ 128 and a lattice density $\rho \in\{16 / 15,16 / 14,16 / 12,16 / 10\}$. Simulations are performed using $K=1000$ multicarrier blocks composed of symbols taken in a quaternary alphabet forming a quadrature phase-shift keying (QPSK) modulation. Four types of generators (or pulse shape filters) are used: square-root-raised cosine (SRRC), rectangular (RECT) as specified for CP-OFDM [13], time-frequency localization (TFL) maximized and out-of-band energy (OBE) minimized [14]. We notice that the SRRC filter is truncated at length of 32 blocks $\left(32 T_{0}\right)$, and the three last pulse-shapes are characterized by an impulse response equal to $T_{0}$ which ensures a lowcomplexity implementation [13].

We start by using SRRC filters with roll-off parameter $0 \leq$ $\alpha \leq 1$ in order to investigate the mean interference power introduced by a FTN signaling over an ideal channel (fig. 2). In figure 2, we plot the theoretical mean interference power given by (16), the simulated interference power (by letting $\sigma_{z}^{2}=0$ ), as well as the theoretical interference for tight frame expressed by (26). For each $\rho$, the minimum value of $\sigma_{i_{p, q}}^{2} / \sigma_{c}^{2}$ is reached below a given value of $\alpha$. In order to explain this result, it is convenient to consider a system based on the dual lattice with a fixed $T_{0}$ parameter: since the bandwidth of a given subcarrier is $(1+\alpha) F_{0}$ the system is orthogonal if $(1+\alpha) F_{0} \leq$ $1 / T_{0}$ or equivalently if $\alpha \leq \rho-1$. In the FTN case, this appropriate choice of $\alpha$ makes $\boldsymbol{g}$ a tight frame. Notice that the suboptimal results obtained when $\alpha$ is close to zero is due to the truncation of the filters. This effect becomes inconspicuous when $\alpha$ increases.

Figure 3 extends the previous observation to several other filters. Since TFL and OBE filters form orthogonal systems in the dual lattice configuration, they lead to tight frames in the FTN case. However, the rectangular short filters which corresponds to CP-OFDM does not generate tight frames since $\check{g} \neq(1 / A) g$. Furthermore, the suboptimal performances of rectangular filters are justified because $\check{\boldsymbol{g}}$ and $\boldsymbol{g}$ are neither canonical dual frames.

Finally, we examine tight frame signaling schemes over an AWGN channel. TFL and SRRC filters $(\alpha=\rho-1)$ are chosen for this simulation. Figure 4 shows the global NMSE of the two filters and the corresponding theoretical values as a function of $\sigma_{c}^{2} / \sigma_{z}^{2}$. We observe that the simulation results perfectly coincide with the theoretical values, the tight frame NMSE equation is thus validated. 


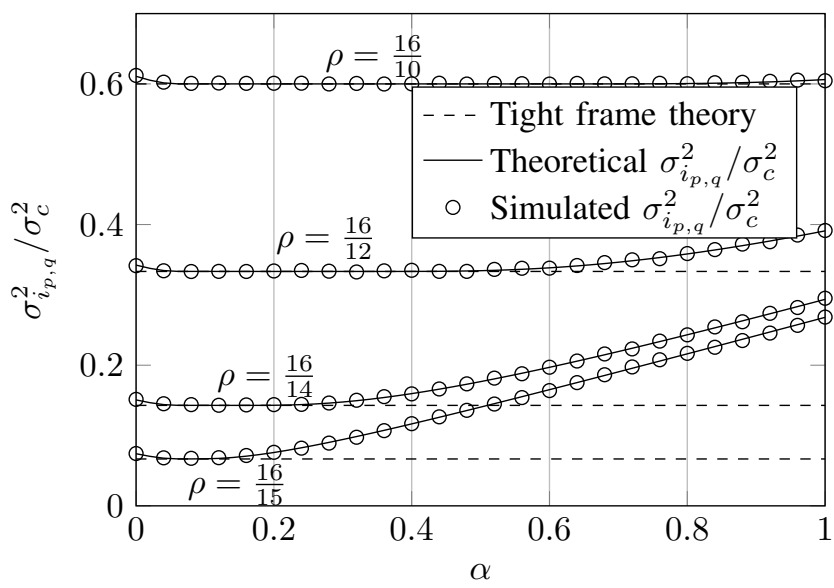

Fig. 2: Mean interference power over an ideal channel using SRRC filters with roll-off factor $\alpha$.

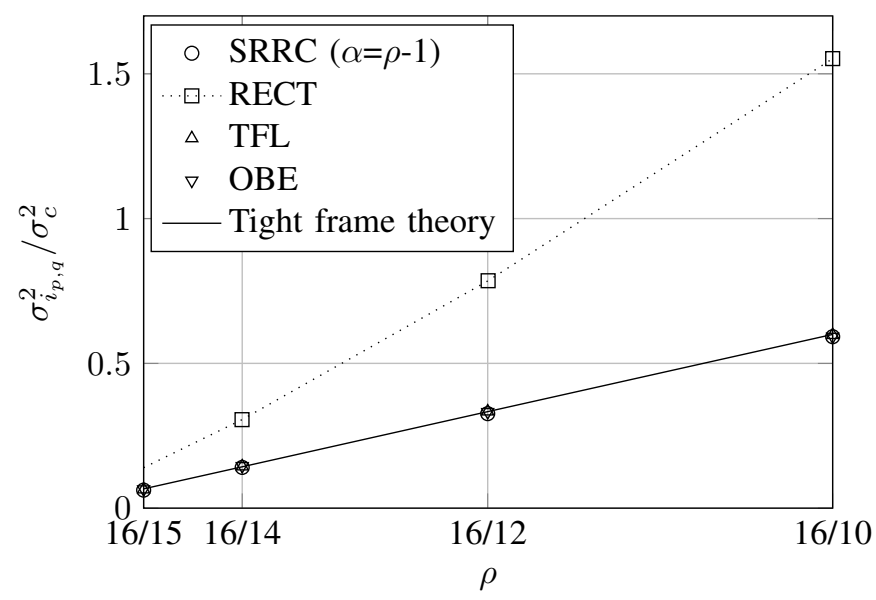

Fig. 3: Mean interference power as a function of the density $\rho$ for various pulse shapes.

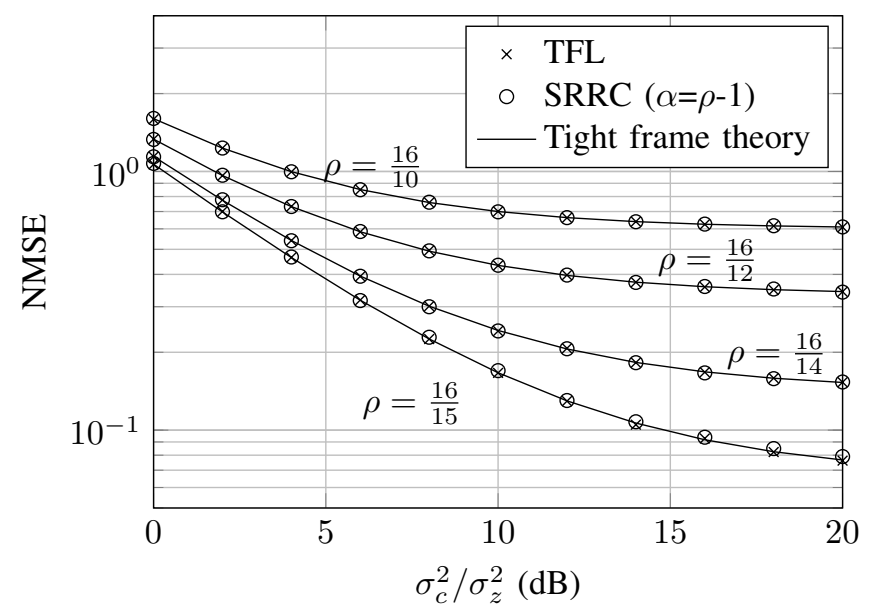

Fig. 4: NMSE performances as a function of $\sigma_{c}^{2} / \sigma_{z}^{2}$ and $\rho$ using tight frames.

\section{CONClusion}

We have expressed the input-output relations of a linear multicarrier transceiver operating at a high signaling density that prevents perfect reconstruction of the symbols. In this "faster-than-Nyquist" context, we have shown several conditions in order to minimize the MSE of such transceivers based on Weyl-Heisenberg frames. First of all, inter-pulse interference is minimized if canonical dual frames are used. Futhermore, the use of tight frames is optimal when a white gaussian noise is added.

Future investigations to be performed on FTN multicarrier signaling consist of designing efficient implementation schemes, including low-complexity equalizers. A performance analysis over time-frequency selective channels would also be interesting in order to target practical applications.

\section{ACKNOWLEDGEMENT}

This work has been supported by Orange-Labs, under a research contract on faster-than-Nyquist signaling.

\section{REFERENCES}

[1] H. Nyquist, "Certain topics in telegraph transmission theory," American Institute of Electrical Engineers, Transactions of the, vol. 47, no. 2, pp. 617-644, 1928.

[2] J. E. Mazo, "Faster than Nyquist signaling," Bell System Technical Journal, vol. 54, pp. 1451-1462, October 1975.

[3] G. Forney, "Maximum-likelihood sequence estimation of digital sequences in the presence of intersymbol interference," Information The ory, IEEE Transactions on, vol. 18, no. 3, pp. 363-378, 1972.

[4] F. Rusek and J. Anderson, "Non binary and precoded faster than Nyquist signaling," Communications, IEEE Transactions on, vol. 56, no. 5, pp $808-817$, may 2008.

[5] A. Prlja, J. Anderson, and F. Rusek, "Receivers for faster-than-Nyquist signaling with and without turbo equalization," in Information Theory, 2008. ISIT 2008. IEEE International Symposium on, july 2008, pp. 464 -468 .

[6] A. Prlja and J. Anderson, "Reduced-complexity receivers for strongly narrowband intersymbol interference introduced by faster-than-Nyquist signaling," Communications, IEEE Transactions on, vol. 60, no. 9, pp. 2591-2601, September 2012

[7] W. Kozek and A. F. Molisch, "Nonorthogonal pulseshapes for multicarrier communications in doubly dispersive channels," IEEE J. Sel. Areas Commun., vol. 16, no. 8, pp. 1579-1589, 1998.

[8] T. Strohmer and S. Beaver, "Optimal OFDM design for time-frequency dispersive channels," IEEE Trans. Commun., vol. 51, no. 7, pp. 1111$1122,2003$.

[9] F. Rusek and J. Anderson, "Multistream faster than Nyquist signaling," Communications, IEEE Transactions on, vol. 57, no. 5, pp. 1329-1340, may 2009.

[10] D. Dasalukunte, F. Rusek, and V. Owall, "Multicarrier faster-thanNyquist transceivers: Hardware architecture and performance analysis," Circuits and Systems I: Regular Papers, IEEE Transactions on, vol. 58, no. 4 , pp. $827-838$, april 2011

[11] O. Christensen, Frames and bases: An introductory course. Birkhauser, 2008.

[12] H. Feichtinger and T. Strohmer, Gabor analysis and algorithms: Theory and applications. Birkhauser, 1998

[13] D. Roque, C. Siclet, and P. Siohan, "A performance comparison of fbmc modulation schemes with short perfect reconstruction filters," in Telecommunications (ICT), 2012 19th International Conference on, April 2012, pp. 1-6.

[14] D. Pinchon and P. Siohan, "Closed-form expressions of optimal short pr fmt prototype filters," in Global Telecommunications Conference (GLOBECOM 2011), 2011 IEEE, Dec 2011, pp. 1-5. 